Review

\title{
Friend or Foe? An Unrecognized Role of Uric Acid in Cancer Development and the Potential Anticancer Effects of Uric Acid-lowering Drugs
}

\author{
Shuyi Mi1,3\#, Liang Gong2\# and Ziqi Sui ${ }^{1,4}{ }^{\boxplus}$ \\ 1. Department of Gastroenterology, The First People's Hospital of Yuhang District, Hangzhou, Zhejiang Province, China. \\ 2. Department of Otolaryngology, Cixi People's Hospital, Ningbo, Zhejiang Province, China. \\ 3. School of Medicine, Zhejiang University City College, Hangzhou, Zhejiang Province, China. \\ 4. Department of Pathophysiology, College of Basic Medical Sciences, Jiamusi University, Jiamusi, Heilongjiang Province, China. \\ \#These authors have contributed equally to this work.
}

$\triangle$ Corresponding author: Dr. Ziqi Sui, Department of Gastroenterology, The First People's Hospital of Yuhang District, 369 Yingbin Road, Hangzhou, Zhejiang 310009, China. Tel.: 86-571-89369161; Fax: 86-571-86224254; E-mail: wenqisui@jmsu.edu.cn.

() The author(s). This is an open access article distributed under the terms of the Creative Commons Attribution License (https://creativecommons.org/licenses/by/4.0/). See http:/ /ivyspring.com/terms for full terms and conditions.

Received: 2020.03.20; Accepted: 2020.06.15; Published: 2020.07.06

\begin{abstract}
In recent years, metabolic syndrome (Mets) has been a hot topic among medical scientists. Mets has an intimate relationship with the incidence and development of various cancers. As a contributory factor of Mets, hyperuricemia actually plays an inseparable role in the formation of various metabolic disorders. Although uric acid is classically considered an antioxidant with beneficial effects, mounting evidence indicates that a high serum uric acid (SUA) level may serve as a pro-oxidant to generate inflammatory reactions and oxidative stress. In this review, we describe the unrecognized role of hyperuricemia in cancer development and summarize major mechanisms linking uric acid to carcinogenesis. Furthermore, we also discuss the potential mechanism of liver metastasis of cancer and list some types of uric acid-lowering agents, which may exert anticancer effects.
\end{abstract}

Key words: hyperuricemia; cancer risk; metabolic syndrome; liver metastasis; uric acid-lowering agents

\section{Introduction}

In recent years, metabolic syndrome (Mets) has been a hot topic among medical scientists. Mets indicates a cluster of metabolic abnormalities including abdominal adiposity, insulin resistance, hyperglycemia, hypertension, and dyslipidemia, causing an increased morbidity and social economic burdens [1]. Increasing evidence has shown that Mets has a close relationship with the incidence and development of some specific cancers, such as breast cancer, ovarian cancer, and pancreatic cancer [2-4]. However, as a contributory factor of Mets, hyperuricemia plays an important role in the formation of various metabolic disorders, including diabetes, obesity, hypertension, and so on $[5,6]$. While it is assumed that Mets and cancer share common underlying mechanisms of oxidative stress and inflammation [4], we have good reason to believe that hyperuricemia is also detrimental to cells, which has not been widely studied in the occurrence and development of cancer.

Hyperuricemia, or increased serum uric acid (SUA) level, is defined as an average SUA level $>6.8$ $\mathrm{mg} / \mathrm{dL}(404 \mu \mathrm{M})$ with or without the recognized complication of gout [7]. With a dual role, uric acid is the end product of purine metabolism via xanthine oxidoreductase, which is mainly eliminated by the kidney and the intestinal tract [8]. When uricemia is in the normal range, with its powerful antioxidative activity, uric acid is thought to scavenge free radicals and contribute to the total antioxidative capacity of plasma [9]. Studies have indicated that uric acid provides a protective role in red blood cells and nerve cells by providing an antioxidant defense [10, 11]. It is also assumed that the radical scavenging action of 
circulating uric acid decreases neoplastic transformation, to reduce the risk of cancer [4]. However, a study has recently reported that the antioxidant activity of uric acid is not as strong as either hydrophilic vitamin $\mathrm{C}$ or hydrophobic vitamin $\mathrm{E}$, and is unlikely to play an important protective role by quenching oxygen radicals [12]. In addition, serum uric acid in very high concentrations may trigger inflammatory stress, and it may also have intracellular pro-oxidative activity $[4,13]$. It is wellknown that a pro-oxidant environment confers a growth advantage to tumor cells, and also influences carcinogenic potential by stimulating specific signaling cascades that regulate cell growth and apoptosis [14].

An increasing number of recent studies have reported a positive correlation between certain types of cancer and Mets, but as the central entity linking Mets to inflammation and cancer, uric acid has been neglected, and studies of the relationship between SUA levels and cancers have been limited. In this review, therefore, we describe the relatively unrecognized role of high serum uric acid in cancer development and metastasis and discuss some of the major mechanisms linking uric acid to carcinogenesis. Because high SUA levels might promote the development of cancer, it is assumed that uric acid-lowering agents are able to exert some benefits in the treatment of cancer. With the increasing resistance to traditional cancer drugs, we believe the use of uric acid-lowering therapy may constitute a novel strategy for the management of some refractory cancers.

\section{Uric acid as a risk factor in various cancers}

Hyperuricemia is the consequence of purine metabolism disorders, which can lead to tumorigenesis and cancer [15]. Increasing numbers of studies have recently suggested that a high level of SUA is associated with higher cancer incidence and mortality [16, 17]. In addition, it was reported that there exists a site bias of digestive organs and urological organs in the relationship between high SUA levels and cancer mortality $[15,18]$.

A statistically significant association was found between higher SUA levels and increased mortality of total cancers, especially the specific sites of digestive cancer, which is also more significant in females than males [18]. After eliminating preclinical diseases, a Chinese study suggested that elevated SUA was independently and positively connected with the risk of digestive cancer and cancer mortality among hypertensive Chinese [19]. Also, a study using mouse model without gene of urate oxidase, has observed that the majority of mice spontaneously developed hepatocellular carcinoma by the age of 2 years [20], which indicates the potential role of hyperuricemia in cancer development. For nasopharyngeal cancer, low post-treatment plasma uric acid levels may be better for patients who benefit from additional aggressive treatment after intensity-modulated radiotherapy, implicating low SUA as possibly conducive to cancer therapy [21]. Evidence has shown that preoperative SUA is an independent prognostic predictor in esophageal squamous cell carcinoma patients who undergo R0 esophagectomy, and patients with a higher SUA level might have significantly shorter 1, 3, and 5 year survival times than patients with a relatively low SUA level [22]. For colorectal cancer $(\mathrm{CRC})$, it was found that SUA levels gradually increased from stage I to stage IV, suggesting that the SUA level reflected the severity of CRC and may help to evaluate the therapy effect as well as the prognoses of CRC patients [14]. In addition, an elevated serum level of uric acid was shown to be a significant prognostic marker for lymphatic metastasis in patients with colon cancer [23]. Among pancreatic cancer patients, it was also observed in a large cohort study that elevated uric acid levels were an independently poor prognostic factor for overall survival [24].

In addition to its association with the development of digestive cancer, SUA levels also correlate with the incidence of urological cancers. It was found that gout patients had a higher risk of prostate cancer, followed by bladder and renal cancers [25]. A Swedish study of males with metabolic syndrome showed that high SUA levels were an independent significant predictor of prostate cancer [26]. It was reported that a postoperative increase of $\geq$ $10 \%$ in the SUA level was predictive of decreased overall survival (OS) and recurrence free survival (RFS) in stage I-III renal cell carcinoma patients, while improved OS and RFS were observed in patients with decreased/stable SUA levels at both 5 and 10 years [27].

It is also not rare for cancer patients, especially patients with lymphocytic leukemia and Burkitt's lymphoma, to have a high SUA level, among whom a high plasma uric acid level may occur as a result of increased purine metabolism by xanthine oxidase as a consequence of tumor cell breakdown [28]. A high SUA level was associated with a poor prognosis in acute myelocytic leukemia patients [29]. It was demonstrated that diffuse, large B-cell lymphoma patients having elevated SUA levels showed consistently worse conditional survival outcomes when compared with patients with lower uric acid levels, with their conditional outcomes only approaching those of the lower uric acid patients 
approximately 5 years after diagnosis [30]. As for respiratory organs, it has been found that among the non-small cell lung cancer patients who had higher SUA levels; there was a higher percentage of brain metastasis, and a shorter time until brain metastasis and lower overall survival [31]. In addition to leading to increased mortality and lower prognosis of cancer patients, hyperuricemia may also reduce the effectiveness of anticancer agents. It has been reported that the elevated SUA levels in hyperuricemia mice also negatively impacted on the effectiveness of immunotherapy to delay growth of melanoma [32].

Gout, defined as a progressive metabolic disease characterized by symptomatic hyperuricemia, is also considered a risk factor for cancer [33, 34]. Additional evidence has shown that gout increases the risk of cancer, and a higher incidence from all causes of cancer has been found in the high prevalence of various gout-related comorbidities [15, 35]. A nationwide population study investigating the relationship between gout and cancer found that the annual incidence of cancer in gout patients was more than double that of the normal population [36]. In another study, SUA $>0.56 \mathrm{mmol} / \mathrm{L}$ and crystalproven gout were found to be strongly associated with mortality and other chronic diseases [37]. Moreover, it was found that a genetically determined lifelong high exposure to urate is more detrimental than high plasma urate, which develops later in life, because lifelong high plasma urate results in a higher risk of cancer and all-cause mortality [16].

\section{The main mechanisms of uric acid during cancer development: Inflammation and oxidative stress}

There is increasing evidence supporting the potential role of uric acid metabolism in carcinogenesis, involving uric acid-induced inflammation and the production of reactive oxygen species (ROS) in the interaction between uric acid and the immune system [38-40]. And it is well known that long-term chronic inflammation in tumor microenvironments has a great impact on neoplasia and tumor progression as well as on immunity, which also indicates the role of uric acid-induced inflammation in cancer development [41, 42].

When patients develop hyperuricemia, uric acid saturates body fluids and undergoes a phase change by nucleating into crystals of monosodium urate (MSU), which are the prime components that trigger symptoms and cause diseases [40]. After its release from dying cells, uric acid is believed to be one of the damage-associated molecular patterns, which alert the immune system to an abnormal situation [43]. As a result, uric acid is detected and leukocytes such as neutrophils and macrophages infiltrate tissues; dendritic cells can also be stimulated, causing acute inflammation [44, 45]. When MSU particles are ingested by phagocytes, they stimulate the NOD-like receptor family, pyrin domain-containing 3 (NLRP3) inflammasomes to activate the caspase- 1 protease, which cleaves IL-1 $\beta$ into its active form, thus eventually producing the proinflammatory form of the IL-1 $\beta$ cytokine [46].

The results of another recent study provide evidence that soluble uric acid (sUA) is also responsible for increasing IL-1 $\beta$ production in an Nlrp3- and Myd88-dependent manner [39]. When cells from healthy subjects were pretreated with uric acid, it specifically downregulated the production of the anti-inflammatory cytokine IL-1 receptor antagonist (IL-1Ra) and, as a result, Toll-like receptorinduced proinflammatory cytokine production was significantly increased and that IL- $1 \beta$ is a signaling molecule secreted when the NLRP3 inflammasome is activated [47]. Furthermore, some studies reported that urate-induced immune programming also plays an important role in immune injury [48]. It is supposed that the upregulation of mRNA levels of inflammasome-related genes like IL-1 $\beta$ and NLRP3 are dependent on sUA production, which indicates sUA is able to alter the transcriptional program of the cell and modulate cytokine production, ultimately leading to the exacerbation of inflammation responses [49]. Also, sUA gives rise to elevated serum chemokine (C-C motif) ligand 2 (CCL2) which is a chemoattractant recruiting circulating monocytes which play a part in chronic low-grade inflammation [50]. Recently a study also observed elevated lipopolysaccharide and tumor necrosis factor- $\alpha$ in hyperuricemia mice, suggesting hyperuricemia mice were in low systemic inflammation [51].

Apart from inflammatory stress, it is also generally thought that ROS induced by uric acid play a central role in carcinogenesis [52]. On the one hand, hyperuricemia mostly results from excessive activity of xanthine oxidase in catalyzing uric acid production, among which also exists excessive ROS production [8]. On the other, it is supposed that uric acid exerts a pro-oxidant activity mainly in an intracellular way. NLRP3 inflammasome stimulation by sUA is accompanied by cellular redox state changes increasing in the mitochondrial area, resulting in increased mitochondrial ROS production [39]. Meanwhile, uric acid crystals activate the immune system, acting as a pro-oxidant molecule that reduces nitric oxide availability, to increase the production of intracellular ROS [53]. 


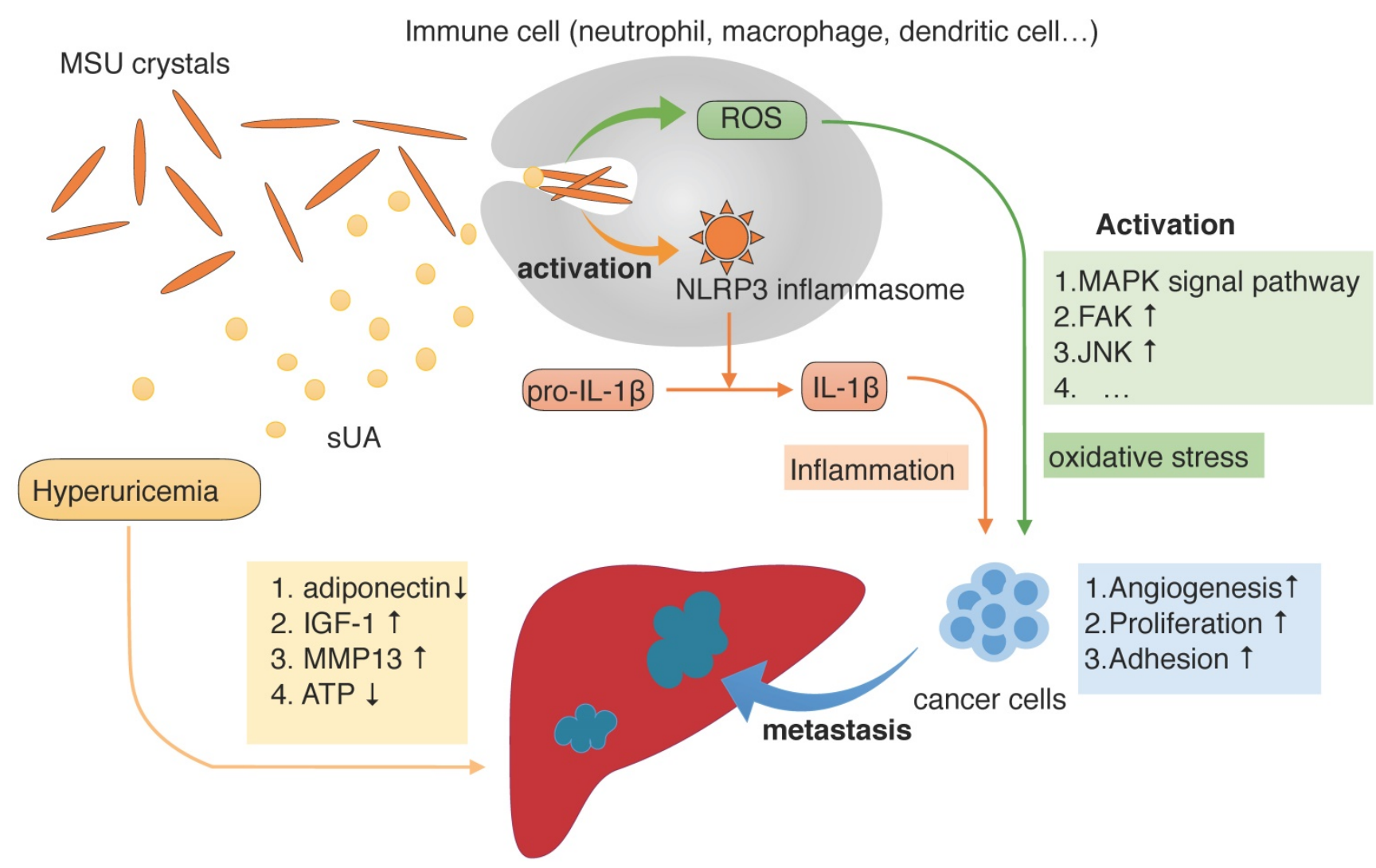

Figure 1. The progression of cancer and metastasis to the liver during hyperuricemia conditions. During hyperuricemia, the main forms of uric acid are soluble uric acid (sUA) and monosodium urate (MSU) crystals. As one of the damage-associated molecular patterns that alert the immune system to injurious situations, MSU crystals are detected and ingested by phagocytes, which stimulate the NOD-like receptor family and pyrin domain containing 3 (NLRP3) inflammasomes and generate reactive oxygen species (ROS) mainly in intracellular way. NLRP3 inflammasomes then promote cleavage of IL-1 $\beta$ into its active form, thus producing the IL-1 $\beta$ proinflammatory cytokine. Depending on the presence of MSU, sUA also participates in this process. The presence of ROS and IL-1 $\beta$ result in oxidative stress and inflammatory responses, respectively, which promote cancer development. Furthermore, hyperuricemia also alters the microenvironment of the liver, making it more likely that cancer will metastasize.

Table 1. The mediators of the development of cancer in the presence of high serum uric acid levels

\begin{tabular}{lll}
\hline Cause & Factor & Cancer development \\
\hline activated NLRP3 inflammasome & Neutrophils, macrophages & Cancer risk $\uparrow$ \\
chronic low-grade inflammation & CCL2 $\rightarrow$ monocytes & LOS $\uparrow$ \\
VEGF, CDK inhibitor, MMPs & ROS $\uparrow$ & Insulin resistance, hypoadiponectinemia, fructose metabolism \\
MMPs, EMK, FAK & & \\
NAFLD &
\end{tabular}

The link between ROS and cancer promotion has been known for many years. It is thought that ROS play a dual role in the regulation of the tumor cell signaling pathway [54]. ROS activate signaling pathways related to proliferation, survival, angiogenesis and metastasis, and promote the occurrence, development, and metastasis of tumors. Alternatively, a high level of ROS can induce cell apoptosis, promoting cell aging, and inhibiting the cell cycle [55]. The potential effects of ROS on oncogenesis include: i) elevated ROS can activate Jun N-terminal kinase (JNK) and p38 mitogen activated-protein kinase (MAPK) signaling, which downregulates cyclins and induces cyclin-dependent kinase $(\mathrm{CDK})$ inhibitors, resulting in cell cycle arrest [56]. ii) Levels of ROS are correlated with the activity of matrix metalloproteinases (MMPs), and increasing MMPs in the tumor microenvironment induce tumor oncogenesis $[57,58]$. iii) ROS induce endothelial cell tube formation and the production of angiogenic factors, such as vascular endothelial growth factor (VEGF) and nitric oxide, which eventually accelerate angiogenesis [59]. iv) ROS activate multiple pathways of the MAPK family to activate receptor tyrosine kinases (RPTKs) to promote the epithelialmesenchymal transition (EMT) and also to create a premetastatic niche in distal organs, establishing a supportive environment for disseminated cancer cells [60]. v) ROS can also activate integrin, induce focal adhesion kinase (FAK) phosphorylation, and promote tumor cell adhesion at the site of metastasis [61]. vi) ROS imbalance leads to protein and lipid oxidation, 
increased mitochondrial membrane permeability, as well as changes of the coupling efficiency of the electron transfer chain, which produces more free radicals and cytochrome $\mathrm{C}$, and activates apoptotic proteases and c-JNK, leading to cell apoptosis [62].

\section{Uric acid-lowering therapy and its ability to lower cancer risk}

Surgical excision is still not effective for most cancers, so chemotherapy is still one of the important comprehensive treatments of cancers. However, with the application of dozens of anticancer or auxiliary anticancer drugs for clinical use, an increasing number of patients become therapeutically resistant. Considering that long-term, costly research is required to develop new drugs, drug repurpose is gradually becoming a novel strategy to treat cancer. The existing drugs like statin and aspirin, which are used to treat cardiovascular diseases, have now received a lot of attention for cancer therapy [63, 64]. Because we now know the potential role of uric acid in cancer initiation and progression, there may also be uric acid-lowering drugs, which have the potential for treating cancer. It has been reported that metabolic remodeling plays an important role in the evolution of some cancers [65], so targeting different mechanisms in carcinogenesis using uric acid is consistent with the findings that uric acid lowering drugs are able to exert anticancer effects.

Microtubules are one of the components of the cytoskeleton, which play an important role in supporting cellular structure. In cancer chemotherapy, drugs that disrupt microtubule dynamics are used widely. Microtubules have long been considered an ideal target for anticancer drugs because of their essential roles in mitosis and formation of the dynamic spindle apparatus [66]. Colchicine, known as an agent used to ameliorate acute gout attacks because of its microtubule disruption activities, has been studied for its possible anticancer effects [67]. By targeting the colchicinebinding site on $\beta$-tubulin, colchicine inhibits microtubule polymerization and leads to prolonged metaphase arrest, thus playing an anti-cancer role as a microtubule inhibitor [68]. In addition, protein expression of rearrangement during transection (RET) was found to correlate with larger tumor size, higher tumor stage, and decreased metastasis-free survival, while colchicine has recently been reported to decrease RET expression by selectively binding RET G-quadruplexes-DNA, which suggests a new mechanism for its anticancer activity [69]. A study has also shown that colchicine induces cell death by apoptosis, and inhibits the invasion and migration of cancer cells by reducing extracellular matrix (ECM) degradation through downregulating MMP9 and the urokinase-type activator system [70]. Actually, in some studies, colchicine in the form of nanoparticles has been used as an anticancer drug to inhibit colon and liver cancer cells [71, 72].

Other commonly used uric acid-lowering agents are xanthine oxidase inhibitors, such as allopurinol and febuxostat. It is known that xanthine oxidase catalyzes the formation of uric acid, as well as the production of ROS [73]. The xanthine oxidase inhibitors are capable of blocking this pathway to reduce the production of uric acid and oxidative stress, which also involves its anticancer activity [74]. It was shown that long-term ( $>1$ year) use of allopurinol resulted in a $34 \%-36 \%$ decrease in the risk of developing prostate cancer [75]. Moreover, allopurinol was reported to attenuate ROS-induced signaling of cytokines, proteolytic activity, and tissue degradation in a rat model of cancer cachexia, which indicated its ability to reduce tissue wasting and improve survival from cancer cachexia [76]. When combined with the tumor necrosis factor-related apoptosis-inducing ligand, allopurinol also strongly induced apoptosis in human hormone-refractory prostate cancer cells [77].

The liver is a frequent site of metastasis for various cancers, and non-alcoholic fatty liver disease (NAFLD) may be a significant factor in the liver microenvironment of cancer metastasis [78, 79]. And uric acid regulates hepatic steatosis and insulin resistance through NLRP3 inflammasomes, playing an important role in the development of NAFLD [80]. While it was also shown that allopurinol reduced uric acid and oxidative stress, therefore decreasing NLRP3 activation and IL-1 $\beta$ levels [81]. Considering its role in attenuating NAFLD, allopurinol may also protect against cancer metastasis to the liver. It has been confirmed that allopurinol had the ability to reduce blood glucose levels and the induction of ROS, and alleviate hepatic oxidative stress, inflammation and steatosis, thus attenuating NAFLD [82]. In the shortterm fructose fed rat model, allopurinol prevented hepatic lipid peroxidation, protein oxidation, and acute adenosine triphosphate (ATP) depletion [83].

As for febuxostat, in addition to its ability to lower levels of serum uric acid, it also has a high affinity for endothelial-bound xanthine oxidase and, therefore, can reduce vascular ROS production, indicating that it may have a strong anticancer potential [84]. Actually, because of its satisfactory efficacy in cancer cell lines, in the study using febuxostat nanoparticles as anticancer drugs for the treatment of lung cancer has suggested it as hopeful strategies [85]. Compared with allopurinol, febuxostat decreased hepatic uric acid levels and xanthine 
oxidase activity in the non-alcoholic steatohepatitis (NASH) mouse model, which was accompanied by more effective prevention of certain features of $\mathrm{NASH}$, including insulin resistance, lipid peroxidation, and liver inflammation, indicating greater efficiency in preventing liver metastasis [86].

\section{Discussion}

In this review, we summarized current knowledge regarding the involvement of hyperuricemia in promoting the progression of various cancers and reducing cancer-related overall survival, which suggested that uric acid might be a potential risk factor of cancer development. Inflammation induced by both sUA and urate crystals as well as ROS production during the interaction between uric acid and immune system are thought to constitute the underlying mechanism stimulating the growth of cancer cells. In the presence of high SUA levels, cancer metastasis is also more likely to occur, which is possibly the consequence of alterations of the organ microenvironment before cancer metastases $[23,31]$.

The liver is a frequent site of metastasis for various cancers because of its unique biological characteristics [78]. To some extent, hyperuricemia may promote the hepatic metastasis of cancer by affecting the liver microenvironment. Sustained exposure to inflammatory stimuli and oxidation induced by uric acid may also lead to the formation of local immunosuppression in the liver, providing a relatively tolerant liver microenvironment that allows the survival and growth of foreign tumor cells [87].

By inducing oxidative stress in both hepatocytes and pancreatic $\beta$, hyperuricemia can result in the development of insulin resistance and growth inhibition, which is associated with ectopic lipid deposition in the liver [88, 89]. And a study showed that increased SUA levels were associated with hypoadiponectinemia, while the lack of adiponectin promoted the progression of hepatic steatosis, fibrosis, and hepatic tumor formation [90]. Alternatively, uric acid can originate from fructose metabolism and when fructose is metabolized, there is a transient decrease in ATP levels, which induces oxidative stress and mitochondrial dysfunction, playing a key role in hepatic steatosis [91, 92]. Because liver steatosis is linked with liver cell injury and inflammation, inflammatory cells such as neutrophils may be recruited during NAFLD, providing a fertile microenvironment for metastasis [79, 87]. MMP13, one of the MMPs, is capable of cleaving various components of the ECM, and adhesion proteins are found to be significantly upregulated in NAFLD and contribute to tumor cell extravasation and establishment of metastases in the liver microenvironment [78]. In addition, elevated circulating insulin-like growth factor (IGF-1) levels produced from a fatty liver promote liver metastasis not only through a direct paracrine effect on tumor cell survival and proliferation but also through indirect effects involving the host microenvironment and proinflammatory responses [93].

It's also worth mentioning that in non-small cell lung cancer patients who had SUA levels over $7.49 \mathrm{mg} / \mathrm{dL}$, the most common organ of metastasis was the brain [31], but the mechanism remains unclear. And the role of hyperuricemia as an independent risk factor for the initiation and progression of cancer is actually still controversial, which it may differ according to sex. It has been suggested that an increased SUA level might be a valuable long-term surrogate marker rather than an independent risk factor or even a carcinogenic substance itself, because increased SUA is also indicative of a lifestyle at increased risk for cancer [94]. A cohort study in Taiwan has reported that uric acid protected against the development of cancer, and it showed that low serum uric acid levels were associated with a higher risk of all cancer mortalities relative to high serum uric acid levels $[95,96]$. In addition, a trend toward a negative association between gout and breast cancer has been reported, while there was a higher risk for male gout patients to develop prostate cancer, and this sex difference in the prevalence of hyperuricemia was correlated with the activity of sex hormones [36]. As a result, additional studies with higher quality are needed to provide a precise determination of the relationship between high SUA and cancer development, particularly with regard to the sex and specific sites of malignancies.

In summary, this review examines the novel idea that uric acid may be an important risk factor for cancer when humans develop a high concentration of SUA. Hyperuricemia may also contribute to the metastasis of some cancers, but the precise mechanism still needs further exploration. We also suggest a new target that may integrate inflammation, oxidative stress, and cell cycle arrest, which have been largely neglected, but are known to be responsive to drug treatment. Based on preliminary clinical evidence, we suggest that drugs that lower serum uric acid might be useful to slow or delay the progression of cancer development. But the specific or approximate extent to which lowering uric acid remains unclear, and recently a cohort study has suggested that an SUA level of $5.7 \mathrm{mg} / \mathrm{dL}(6 \mathrm{mg} / \mathrm{dL}$ in males and $4 \mathrm{mg} / \mathrm{dL}$ in females) is considered safe with respect to mortality [97]. Repurposing these existing drugs may therefore be a novel strategy for 
management of some refractory cancers, but the application of those drugs needs further analyses.

\section{Abbreviations}

CCL2: chemokine (C-C motif) ligand 2; CRC: colorectal cancer; DAMPs: damage-associated molecular patterns; ECM: extracellular matrix; FAK: focal adhesion kinase; EMT: epithelial-mesenchymal transition; MAPK: mitogen activated-protein kinase; Mets: metabolic syndrome; MMPs: matrix metalloproteinases; MSU: monosodium urate; IGF-1: insulin-like growth factor; IL-1Ra: IL-1 receptor antagonist; JNK: Jun N-terminal kinase; NAFLD: non-alcoholic fatty liver disease; NASH: non-alcoholic steatohepatitis; OS: overall survival; RET: rearrangement during transection; RFS: recurrence free survival; ROS: reactive oxygen species; RPTKs: receptor tyrosine kinases; SUA: serum uric acid; sUA: soluble uric acid; XOR: xanthine oxidoreductase; VEGF: vascular endothelial growth factor.

\section{Acknowledgements}

This work was supported by grant from the Health and Family Planning Commission of Zhejiang Province (No. 2015RCB026).

\section{Competing Interests}

The authors have declared that no competing interest exists.

\section{References}

1. Yadav D, Lee ES, Kim HM, Choi E, Lee EY, Lim JS, et al. Prospective study of serum uric acid levels and incident metabolic syndrome in a Korean rural cohort. Atherosclerosis. 2015; 241: 271-7.

2. Cicero AFG, Fogacci F, Giovannini M, Grandi E, Rosticci M, D'Addato S, et al. Serum uric acid predicts incident metabolic syndrome in the elderly in an analysis of the Brisighella Heart Study. Sci Rep. 2018; 8: 11529.

3. Park SK, Oh CM, Kim MH, Ha E, Choi YS, Ryoo JH. Metabolic syndrome, metabolic components, and their relation to the risk of pancreatic cancer. Cancer. 2020

4. Battelli MG, Bortolotti M, Polito L, Bolognesi A. Metabolic syndrome and cancer risk: The role of xanthine oxidoreductase. Redox Biol. 2019; 21: 101070.

5. Guarda NS, Bollick YS, de Carvalho JAM, Premaor MO, Comim FV, Moresco RN. High Serum Uric Acid Is Associated with Tubular Damage and Kidney Inflammation in Patients with Type 2 Diabetes. Dis Markers. 2019; 2019: 6025804.

6. Kanbay M, Jensen T, Solak Y, Le M, Roncal-Jimenez C, Rivard C, et al. Uric acid in metabolic syndrome: From an innocent bystander to a central player. Eur J Intern Med. 2016; 29: 3-8.

7. Ben Salem C, Slim R, Fathallah N, Hmouda H. Drug-induced hyperuricaemia and gout. Rheumatology (Oxford). 2017; 56: 679-88.

8. Battelli MG, Bortolotti M, Polito L, Bolognesi A. The role of xanthine oxidoreductase and uric acid in metabolic syndrome. Biochim Biophys Acta Mol Basis Dis. 2018; 1864: 2557-65.

9. Schlesinger N, Brunetti L. Beyond urate lowering: Analgesic and anti-inflammatory properties of allopurinol. Semin Arthritis Rheum. 2019.

10. Song Y, Tang L, Han J, Gao Y, Tang B, Shao M, et al. Uric Acid Provides Protective Role in Red Blood Cells by Antioxidant Defense: A Hypothetical Analysis. Oxid Med Cell Longev. 2019; 2019: 3435174.

11. Black CN, Bot M, Scheffer PG, Snieder H, Penninx B. Uric acid in major depressive and anxiety disorders. J Affect Disord. 2018; 225: 684-90.
12. Liu D, Yun $Y$, Yang D, Hu X, Dong X, Zhang N, et al. What Is the Biological Function of Uric Acid? An Antioxidant for Neural Protection or a Biomarker for Cell Death. Dis Markers. 2019; 2019: 4081962.

13. Shi Y. Caught red-handed: uric acid is an agent of inflammation. J Clin Invest. 2010; 120: 1809-11.

14. Mao L, Guo C, Zheng S. Elevated urinary 8-oxo-7,8-dihydro-2'deoxyguanosine and serum uric acid are associated with progression and are prognostic factors of colorectal cancer. Onco Targets Ther. 2018; 11: 5895-902.

15. Wang W, Xu D, Wang B, Yan S, Wang X, Yin Y, et al. Increased Risk of Cancer in relation to Gout: A Review of Three Prospective Cohort Studies with 50,358 Subjects. Mediators Inflamm. 2015; 2015: 680853.

16. Kobylecki CJ, Afzal S, Nordestgaard BG. Plasma Urate, Cancer Incidence, and All-Cause Mortality: A Mendelian Randomization Study. Clin Chem. 2017; 63: 1151-60.

17. Deng Z, Gu Y, Hou X, Zhang L, Bao Y, Hu C, et al. Association between uric acid, cancer incidence and mortality in patients with type 2 diabetes: Shanghai diabetes registry study. Diabetes Metab Res Rev. 2016; 32: 325-32.

18. Yan S, Zhang P, Xu W, Liu Y, Wang B, Jiang T, et al. Serum Uric Acid Increases Risk of Cancer Incidence and Mortality: A Systematic Review and Meta-Analysis. Mediators Inflamm. 2015; 2015: 764250.

19. Yang J, Wang Y, Zhao Q, Zhang X, Wang X, Qin X, et al. Association of serum uric acid with increased risk of cancer among hypertensive Chinese. Int J Cancer. 2017; 141: 112-20.

20. Stevenson WS, Hyland CD, Zhang JG, Morgan PO, Willson TA, Gill A, et al. Deficiency of 5-hydroxyisourate hydrolase causes hepatomegaly and hepatocellular carcinoma in mice. Proc Natl Acad Sci U S A. 2010; 107: 16625-30.

21. Lin H, Lin HX, Ge N, Wang HZ, Sun R, Hu WH. Plasma uric acid and tumor volume are highly predictive of outcome in nasopharyngeal carcinoma patients receiving intensity modulated radiotherapy. Radiat Oncol. 2013; 8: 121.

22. Chen YF, Li Q. Prognostic value of pre-operative serum uric acid levels in esophageal squamous cell carcinoma patients who undergo R0 esophagectomy. Cancer Biomark. 2016; 17: 89-96.

23. Yuan $\mathrm{C}, \mathrm{Xu} \mathrm{XH}$, Wang $\mathrm{XL}, \mathrm{Xu} \mathrm{L}$, Chen Z, Li YQ. Relationship between serum uric acid and metastatic and nonmetastatic rectal cancer patients with undergoing no chemotherapy. Medicine (Baltimore). 2016; 95: e5463.

24. Stotz M, Szkandera J. Evaluation of uric acid as a prognostic blood-based marker in a large cohort of pancreatic cancer patients. PLoS One. 2014; 9: e104730.

25. Chen CJ, Yen JH, Chang SJ. Gout patients have an increased risk of developing most cancers, especially urological cancers. Scand J Rheumatol. 2014; 43: 385-90.

26. Hammarsten J, Damber JE, Peeker R, Mellstrom D, Hogstedt B. A higher prediagnostic insulin level is a prospective risk factor for incident prostate cancer. Cancer Epidemiol. 2010; 34: 574-9.

27. Yim K, Bindayi A, McKay R, Mehrazin R, Raheem OA, Field C, et al. Rising Serum Uric Acid Level Is Negatively Associated with Survival in Renal Cell Carcinoma. Cancers (Basel). 2019; 11.

28. Fini MA, Monks J, Farabaugh SM, Wright RM. Contribution of xanthine oxidoreductase to mammary epithelial and breast cancer cell differentiation in part modulates inhibitor of differentiation-1. Mol Cancer Res. 2011; 9: 1242-54.

29. Yamauchi T, Negoro E, Lee S, Takai M, Matsuda Y, Takagi K, et al. A high serum uric acid level is associated with poor prognosis in patients with acute myeloid leukemia. Anticancer Res. 2013; 33: 3947-51.

30. Prochazka KT, Melchardt T, Posch F, Schlick K, Deutsch A, Beham-Schmid $\mathrm{C}$, et al. NCCN-IPI score-independent prognostic potential of pretreatment uric acid levels for clinical outcome of diffuse large B-cell lymphoma patients. Br J Cancer. 2016; 115: 1264-72.

31. Tanriverdi O, Cokmert S, Oktay E, Pilanci KN, Menekse S, Kocar M, et al. Prognostic significance of the baseline serum uric acid level in non-small cell lung cancer patients treated with first-line chemotherapy: a study of the Turkish Descriptive Oncological Researches Group. Med Oncol. 2014; 31: 217

32. Baey C, Yang J, Ronchese F, Harper JL. Hyperuricaemic UrahPlt2/Plt2 mice show altered $\mathrm{T}$ cell proliferation and defective tumor immunity after local immunotherapy with Poly I:C. PLoS One. 2018; 13: e0206827.

33. Petrick JL, Yang B, Altekruse SF, Van Dyke AL, Koshiol J, Graubard BI, et al. Risk factors for intrahepatic and extrahepatic cholangiocarcinoma in the United States: A population-based study in SEER-Medicare. PLoS One. 2017; 12: e0186643.

34. Brook RA, Forsythe A, Smeeding JE, Lawrence Edwards N. Chronic gout: epidemiology, disease progression, treatment and disease burden. Curr Med Res Opin. 2010; 26: 2813-21. 
35. Huang CF, Liu JC, Huang HC, Chuang SY, Chen CI, Lin KC. Longitudinal transition trajectory of gouty arthritis and its comorbidities: a population-based study. Rheumatol Int. 2017; 37: 313-22.

36. Kuo CF, Luo SF, See LC, Chou IJ, Fang YF, Yu KH. Increased risk of cancer among gout patients: a nationwide population study. Joint Bone Spine. 2012; 79: 375-8.

37. Disveld IJM, Zoakman S, Jansen T, Rongen GA, Kienhorst LBE, Janssens $\mathrm{H}$, et al. Crystal-proven gout patients have an increased mortality due to cardiovascular diseases, cancer, and infectious diseases especially when having tophi and/or high serum uric acid levels: a prospective cohort study. Clin Rheumatol. 2019; 38: 1385-91.

38. Gasse P, Riteau N. Uric acid is a danger signal activating NALP3 inflammasome in lung injury inflammation and fibrosis. Am J Respir Crit Care Med. 2009; 179: 903-13.

39. Braga TT, Forni MF, Correa-Costa M, Ramos RN, Barbuto JA, Branco P, et al. Soluble Uric Acid Activates the NLRP3 Inflammasome. Sci Rep. 2017; 7: 39884.

40. Rock KL, Kataoka H, Lai JJ. Uric acid as a danger signal in gout and its comorbidities. Nat Rev Rheumatol. 2013; 9: 13-23.

41. Ahechu P, Zozaya G, Marti P, Hernandez-Lizoain JL, Baixauli J, Unamuno $X$, et al. NLRP3 Inflammasome: A Possible Link Between Obesity-Associated Low-Grade Chronic Inflammation and Colorectal Cancer Development. Front Immunol. 2018; 9: 2918.

42. Iyengar NM, Hudis CA, Dannenberg AJ. Obesity and cancer: local and systemic mechanisms. Annu Rev Med. 2015; 66: 297-309.

43. Weigt SS, Palchevskiy V, Belperio JA. Inflammasomes and IL-1 biology in the pathogenesis of allograft dysfunction. J Clin Invest. 2017; 127: 2022-9.

44. Kataoka H, Yang K, Rock KL. The xanthine oxidase inhibitor Febuxostat reduces tissue uric acid content and inhibits injury-induced inflammation in the liver and lung. Eur J Pharmacol. 2015; 746: 174-9.

45. Gallo PM, Gallucci S. The dendritic cell response to classic, emerging, and homeostatic danger signals. Implications for autoimmunity. Front Immunol. 2013; 4: 138.

46. Shi Y, Mucsi AD, Ng G. Monosodium urate crystals in inflammation and immunity. Immunol Rev. 2010; 233: 203-17.

47. Crisan TO, Cleophas MC, Oosting M, Lemmers H, Toenhake-Dijkstra H, Netea MG, et al. Soluble uric acid primes TLR-induced proinflammatory cytokine production by human primary cells via inhibition of IL-1Ra. Ann Rheum Dis. 2016; 75: 755-62.

48. Joosten LAB, Crisan TO, Bjornstad P, Johnson RJ. Asymptomatic hyperuricaemia: a silent activator of the innate immune system. Nat Rev Rheumatol. 2020; 16: 75-86.

49. Cabau G, Crisan TO, Kluck V, Popp RA, Joosten LAB. Urate-induced immune programming: Consequences for gouty arthritis and hyperuricemia. Immunol Rev. 2020; 294: 92-105.

50. Grainger R, McLaughlin RJ, Harrison AA, Harper JL. Hyperuricaemia elevates circulating CCL2 levels and primes monocyte trafficking in subjects with inter-critical gout. Rheumatology (Oxford). 2013; 52: 1018-21.

51. Xu D, Lv Q, Wang X, Cui X, Zhao P, Yang X, et al. Hyperuricemia is associated with impaired intestinal permeability in mice. Am J Physiol Gastrointest Liver Physiol. 2019; 317: G484-G92.

52. Fini MA, Elias A, Johnson RJ, Wright RM. Contribution of uric acid to cancer risk, recurrence, and mortality. Clin Transl Med. 2012; 1: 16

53. So A, Thorens B. Uric acid transport and disease. J Clin Invest. 2010; 120: 1791-9.

54. Snezhkina AV, Kudryavtseva AV, Kardymon OL, Savvateeva MV, Melnikova NV, Krasnov GS, et al. ROS Generation and Antioxidant Defense Systems in Normal and Malignant Cells. Oxid Med Cell Longev. 2019; 2019: 6175804.

55. Prasad S, Gupta SC, Pandey MK, Tyagi AK, Deb L. Oxidative Stress and Cancer: Advances and Challenges. Oxid Med Cell Longev. 2016; 2016: 5010423.

56. Jiang $Y$, Wang $X, H u$ D. Furanodienone induces G0/G1 arrest and causes apoptosis via the ROS/MAPKs-mediated caspase-dependent pathway in human colorectal cancer cells: a study in vitro and in vivo. Cell Death Dis. 2017; 8: e2815.

57. Burlaka AP, Ganusevich II, Gafurov MR, Lukin SM, Sidorik EP. Stomach Cancer: Interconnection between the Redox State, Activity of MMP-2, MMP-9 and Stage of Tumor Growth. Cancer Microenviron. 2016; 9: 27-32.

58. Holmberg C, Ghesquiere B, Impens F, Gevaert K, Kumar JD, Cash N, et al. Mapping proteolytic processing in the secretome of gastric cancer-associated myofibroblasts reveals activation of MMP-1, MMP-2, and MMP-3. J Proteome Res. 2013; 12: 3413-22.
59. Prasad S, Gupta SC, Tyagi AK. Reactive oxygen species (ROS) and cancer: Role of antioxidative nutraceuticals. Cancer Lett. 2017; 387: 95-105.

60. Liao Z, Chua D, Tan NS. Reactive oxygen species: a volatile driver of field cancerization and metastasis. Mol Cancer. 2019; 18: 65.

61. Galadari S, Rahman A, Pallichankandy S, Thayyullathil F. Reactive oxygen species and cancer paradox: To promote or to suppress? Free Radic Biol Med. 2017; 104: 144-64.

62. Moloney JN, Cotter TG. ROS signalling in the biology of cancer. Semin Cell Dev Biol. 2018; 80: 50-64.

63. Akinwunmi B, Vitonis AF, Titus L, Terry KL, Cramer DW. Statin therapy and association with ovarian cancer risk in the New England Case Control (NEC) study. Int J Cancer. 2019; 144: 991-1000.

64. Hua H, Zhang H, Kong Q, Wang J, Jiang Y. Complex roles of the old drug aspirin in cancer chemoprevention and therapy. Med Res Rev. 2019; 39: 114-45.

65. Chang L, Fang S, Gu W. The Molecular Mechanism of Metabolic Remodeling in Lung Cancer. J Cancer. 2020; 11: 1403-11.

66. Risinger AL, Giles FJ, Mooberry SL. Microtubule dynamics as a target in oncology. Cancer Treat Rev. 2009; 35: 255-61.

67. Kumar A. Potential anticancer role of colchicine-based derivatives: an overview. Anticancer Drugs. 2017; 28: 250-62.

68. Fong A, Durkin A, Lee H. The Potential of Combining Tubulin-Targeting Anticancer Therapeutics and Immune Therapy. Int J Mol Sci. 2019; 20.

69. Wang F, Wang C, Liu Y, Lan W, Han H, Wang R, et al. Colchicine selective interaction with oncogene RET G-quadruplex revealed by NMR. Chem Commun (Camb). 2020; 56: 2099-102.

70. Cho JH, Joo YH, Shin EY, Park EJ, Kim MS. Anticancer Effects of Colchicine on Hypopharyngeal Cancer. Anticancer Res. 2017; 37: 6269-80.

71. Cauda V, Engelke H, Sauer A, Arcizet D, Brauchle C, Radler J, et al. Colchicine-loaded lipid bilayer-coated $50 \mathrm{~nm}$ mesoporous nanoparticles efficiently induce microtubule depolymerization upon cell uptake. Nano Lett. 2010; 10: 2484-92.

72. AbouAitah K, Hassan HA, Swiderska-Sroda A, Gohar L, Shaker OG, Wojnarowicz I, et al. Targeted Nano-Drug Delivery of Colchicine against Colon Cancer Cells by Means of Mesoporous Silica Nanoparticles. Cancers (Basel). 2020; 12.

73. Battelli MG, Polito L, Bortolotti M, Bolognesi A. Xanthine oxidoreductase in cancer: more than a differentiation marker. Cancer Med. 2016; 5: 546-57.

74. Li Y. Discovery of Novel Allopurinol Derivatives with Anticancer Activity and Attenuated Xanthine Oxidase Inhibition. Molecules. 2016; 21.

75. Shih HJ. Long-term allopurinol use decreases the risk of prostate cancer in patients with gout: a population-based study. Prostate Cancer Prostatic Dis. 2017; 20: 328-33.

76. Springer J. Inhibition of xanthine oxidase reduces wasting and improves outcome in a rat model of cancer cachexia. Int J Cancer. 2012; 131: 2187-96.

77. Yasuda T, Yoshida T, Goda AE, Horinaka M, Yano K, Shiraishi T, et al. Anti-gout agent allopurinol exerts cytotoxicity to human hormone-refractory prostate cancer cells in combination with tumor necrosis factor-related apoptosis-inducing ligand. Mol Cancer Res. 2008; 6: $1852-60$.

78. Mendonsa AM, VanSaun MN, Ustione A, Piston DW, Fingleton BM, Gorden DL. Host and tumor derived MMP13 regulate extravasation and establishment of colorectal metastases in the liver. Mol Cancer. 2015; 14: 49.

79. Kondo T, Okabayashi K, Hasegawa H, Tsuruta M, Shigeta K, Kitagawa $Y$. The impact of hepatic fibrosis on the incidence of liver metastasis from colorectal cancer. Br J Cancer. 2016; 115: 34-9.

80. Wan $X, X u$ C, Lin Y, Lu C, Li D, Sang J, et al. Uric acid regulates hepatic steatosis and insulin resistance through the NLRP3 inflammasome-dependent mechanism. J Hepatol. 2016; 64: 925-32.

81. Andrade-Oliveira V, Foresto-Neto O, Watanabe IKM, Zatz R, Camara NOS. Inflammation in Renal Diseases: New and Old Players. Front Pharmacol. 2019; 10: 1192.

82. Wang W, Wang C, Ding XQ, Pan Y, Gu TT, Wang MX, et al. Quercetin and allopurinol reduce liver thioredoxin-interacting protein to alleviate inflammation and lipid accumulation in diabetic rats. Br J Pharmacol. 2013; 169: 1352-71.

83. Garcia-Arroyo FE, Monroy-Sanchez F, Munoz-Jimenez I, Gonzaga G, Andres-Hernando A, Zazueta C, et al. Allopurinol Prevents the Lipogenic Response Induced by an Acute Oral Fructose Challenge in Short-Term Fructose Fed Rats. Biomolecules. 2019; 9.

84. Tausche AK, Christoph M, Forkmann M, Richter U, Kopprasch S, Bielitz $\mathrm{C}$, et al. As compared to allopurinol, urate-lowering therapy with febuxostat has superior effects on oxidative stress and pulse wave 
velocity in patients with severe chronic tophaceous gout. Rheumatol Int. 2014; 34: 101-9.

85. Alfaifi MY, Shati AA, Elbehairi SEI, Fahmy UA, Alhakamy NA, Md S. Anti-tumor effect of PEG-coated PLGA nanoparticles of febuxostat on A549 non-small cell lung cancer cells. 3 Biotech. 2020; 10: 133.

86. Nishikawa T, Nagata N, Shimakami T, Shirakura T, Matsui C, Ni Y, et al. Xanthine oxidase inhibition attenuates insulin resistance and diet-induced steatohepatitis in mice. Sci Rep. 2020; 10: 815.

87. Lv Y, Patel N, Zhang HJ. The progress of non-alcoholic fatty liver disease as the risk of liver metastasis in colorectal cancer. Expert Rev Gastroenterol Hepatol. 2019; 13: 1169-80.

88. Samuel VT, Shulman GI. Nonalcoholic Fatty Liver Disease, Insulin Resistance, and Ceramides. N Engl J Med. 2019; 381: 1866-9.

89. Mosca A, Nobili V, De Vito R, Crudele A, Scorletti E, Villani A, et al. Serum uric acid concentrations and fructose consumption are independently associated with NASH in children and adolescents. J Hepatol. 2017; 66: 1031-6.

90. Yang $\mathrm{Q}, \mathrm{Fu} \mathrm{C}$, Xiao J, Ye Z. Uric acid upregulates the adiponectinadiponectin receptor 1 pathway in renal proximal tubule epithelial cells. Mol Med Rep. 2018; 17: 3545-54.

91. Jensen T, Abdelmalek MF, Sullivan S, Nadeau KJ, Green M, Roncal C, et al. Fructose and sugar: A major mediator of non-alcoholic fatty liver disease. J Hepatol. 2018; 68: 1063-75.

92. Lanaspa MA, Sanchez-Lozada LG, Choi YJ, Cicerchi C, Kanbay M, Roncal-Jimenez CA, et al. Uric acid induces hepatic steatosis by generation of mitochondrial oxidative stress: potential role in fructose-dependent and -independent fatty liver. J Biol Chem. 2012; 287: 40732-44.

93. Wu Y, Brodt P, Sun H, Mejia W, Novosyadlyy R, Nunez N, et al. Insulin-like growth factor-I regulates the liver microenvironment in obese mice and promotes liver metastasis. Cancer Res. 2010; 70: 57-67.

94. Strasak AM, Lang S, Kneib T, Brant LJ, Klenk J, Hilbe W, et al. Use of penalized splines in extended Cox-type additive hazard regression to flexibly estimate the effect of time-varying serum uric acid on risk of cancer incidence: a prospective, population-based study in 78,850 men. Ann Epidemiol. 2009; 19: 15-24.

95. Kuo CF, See LC, Yu KH, Chou IJ, Chiou MJ, Luo SF. Significance of serum uric acid levels on the risk of all-cause and cardiovascular mortality. Rheumatology (Oxford). 2013; 52: 127-34.

96. Taghizadeh N, Vonk JM, Boezen HM. Serum uric acid levels and cancer mortality risk among males in a large general population-based cohort study. Cancer Causes Control. 2014; 25: 1075-80.

97. Hu L, Hu G, Xu BP, Zhu L, Zhou W, Wang T, et al. U-Shaped Association of Serum Uric Acid With All-Cause and Cause-Specific Mortality in US Adults: A Cohort Study. J Clin Endocrinol Metab. 2020; 105. 